\title{
Transport phenomena of inorganic-organic cation exchange nanocomposite membrane: a comparative study with different methods
}

\author{
Md. Ramir Khan · Rafiuddin
}

Received: 12 January 2014/ Accepted: 6 March 2014/Published online: 3 April 2014

(c) The Author(s) 2014. This article is published with open access at Springerlink.com

\begin{abstract}
Transport studies have been carried out across a newly synthesized inorganic-organic nanocomposite membrane. Membrane potential measurements have been measured in various monovalent electrolyte environments at different concentrations to analyse the relationship between transport properties and fixed charge concentration of the membrane. The charge densities were found to follow the order $\mathrm{K}^{+}>\mathrm{Na}^{+}>\mathrm{Li}^{+}$. The counter-ion transport number, ionic mobility ratio and permselectivity of the nanocomposite membrane have also been calculated which reveal that the inorganic-organic nanocomposite membrane shows a higher selectivity towards $\mathrm{K}^{+}$ions; however, the selectivity decreases with decrease in dilution for all monovalent electrolytes tested. These membranes were comprehensively characterized for their physicochemical properties, morphology, molecular interactions and crystallinity by sophisticated techniques. The ionexchange capacity, volume void porosity and water uptake of the membrane were found to depend on the polystyrene content in the membrane phase and these properties decreased with increase in the amount of polymer (15-35\% by mass). Moreover, membrane containing $25 \%$ of polystyrene exhibited good selectivity along with moderate ion-exchange capacity, which may be used for their application in electro-driven separation at high temperatures or for other electrochemical processes.
\end{abstract}

Md. Ramir Khan $(\bowtie) \cdot$ Rafiuddin

Membrane Research Laboratory, Physical Chemistry Division, Department of Chemistry, Aligarh Muslim University,

Aligarh 202002, India

e-mail: rami1210amualig@gmail.com
Keywords Cobalt carbonate nanocomposite $\cdot$ Transport number - Permselectivity - Physicochemical properties . Tasaka, and Kobatake methods · Fixed charge density

\section{List of symbols and abbreviations}

$c_{1}, c_{2}$ Concentrations $(\mathrm{mol} / \mathrm{L})$ of the monovalent electrolyte in the lower and higher concentration sides of the membrane, respectively

C Average electrolyte concentration ( $\mathrm{mol} / \mathrm{L})$

SEM Scanning electron microscope

XRD X-ray diffraction

$D \quad$ Crystallite size

$\beta \quad$ Full width at half-maximum (FWHM)

$\theta \quad$ Diffraction angle $\left({ }^{\circ}\right)$

$W_{c}^{\mathrm{m}} \quad$ Water content in terms of water concentration $(\mathrm{mol} / \mathrm{L})$

$w_{\mathrm{w}} \quad$ Wet membrane weight $(\mathrm{g})$

$w_{\mathrm{d}} \quad$ Dry membrane weight $(\mathrm{g})$

$\tau_{\mathrm{m}} \quad$ Volume void porosity of the membrane

$\rho_{\mathrm{w}} \quad$ Density of water $\left(\mathrm{g} / \mathrm{cm}^{3}\right)$

$\rho_{\mathrm{d}} \quad$ Density of the dry membrane

$\psi_{\mathrm{m}} \quad$ Membrane potential (mV)

$t_{\text {-app }} \quad$ Transparent transport number of anion

$t_{+}^{\mathrm{m}} \quad$ Counter-ion transport number in the membrane phase

$t_{+} \quad$ Transport number of the cation in the solution phase

\section{Introduction}

The development of new membrane materials has tremendously gained the advantage of an interdisciplinary approach integrating recent advances in the field of material science in the past few decades. Membranes fulfill a 
large number of complex mass and energy transport functions very efficiently and with minimal energy expenditure. They can transport individual chemical components selectively and under certain conditions, which may be controlled by specific components such as an electrical potential gradient.

The structure and chemical nature or electrical charges of the membrane material along with the properties of the permeating component such as its size, its chemical nature, or electrical charge, and forces such as concentration, pressure, or electrical potential gradients across the membrane determine the transport rate of a component through it. The charges developed across the membranes play a significant role in the sorption and transport of simple electrolytes and provide several vital electrochemical properties not only to synthetic membranes but also to natural membranes [1]. One of the most important properties imparted by such charges is the difference in the permeabilities of co-ions, counter-ions and neutral molecules.

Membrane potential measurement is one of the electrochemical methods applied to evaluate the effect of charges of membranes on their transport properties arising due to electrolyte concentration. Membrane potential is the algebraic sum of diffusion potential and two Donnan potentials, determined by the partition of ions into the pore as well as the mobilities of ions within the membrane phase with respect to the external phase $[2,3]$. The transport of ions across the membrane can be related to the membrane potential caused by concentration gradient of an electrolyte impressed upon the membrane. The present paper reports the synthesis and transport properties of polystyrene blended cobalt carbonate membrane. The inorganic-organic composite membrane has been studied because such membranes are a promising and fast emerging research area with various remarkable importances arising due to the synergetic properties of both the inorganic and organic components [4-7]. The transport studies of the newly synthesized membrane have been carried out using various methods suggested by different research groups [8-10] and the fixed charge density values have been compared to check the applicability of the theories to the membrane system.

\section{Experimental section}

\section{Reagents and solutions}

Cobalt (II) chloride (from E. Merck (India) Limited, Mumbai, $97 \%$ purity) and sodium carbonate (99.9\% purity, Fine Chemicals Division, Mumbai) had been used for the synthesis of the inorganic membrane. Deionised water (water purification systems, integrate; with reverse osmosis (RO) conductivity $0-200 \mu \mathrm{s} / \mathrm{cm}$ ) was used to prepare all solutions of the reagents, which are of analytical grade. Pure sodium chloride, potassium chloride and ammonium chloride were obtained from E. Merck (India) Limited while lithium chloride from Loba - Chemie Indoaustranal Co. (India). The working solutions of the electrolytes $\left(\mathrm{KCl}, \mathrm{NaCl}, \mathrm{LiCl}\right.$ and $\left.\mathrm{NH}_{4} \mathrm{Cl}\right)$ of the required concentrations were prepared by appropriately diluting their stock solutions.

Synthesis of polystyrene blended cobalt carbonate membranes

The white precipitates of cobalt carbonate have been synthesized by sol-gel method by reacting $0.25 \mathrm{M}$ cobalt (II) chloride solution with $0.25 \mathrm{M}$ solution of sodium carbonate [11]. The resulting mixture is stirred well with a magnetic bar keeping the temperature constant at $80{ }^{\circ} \mathrm{C}$ for $24 \mathrm{~h}$ until one-phase solution is formed that goes through a solution-to-gel transition. The mixture was adjusted to $\mathrm{pH}$ 2 by adding diluted $\mathrm{HCl}$ solution. The precipitates were kept overnight in the mother liquor for digestion. After decanting off the supernatant liquid, the remaining precipitates were washed with demineralised water to remove any excess reagent of electrolytes and then dried at $100 \pm 1{ }^{\circ} \mathrm{C}$ for another $24 \mathrm{~h}$. These were powdered and sieved through a 200 mesh (Granule size $<0.07 \mathrm{~mm}$ ). Pure crystalline polystyrene was also ground to fine powder and sieved through a 200 mesh. The inorganic precipitates were then mixed with granulated polystyrene with the help of a pestle and mortar to get ion-exchange membranes having a varying percentage (15-35\%) of polystyrene by mass. Such membranes were used to understand the changes in their physicochemical behaviour as a function of the quantity of polystyrene that is blended with the inorganic component while membranes having $25 \%$ polystyrene were only selected for transport studies. A flowchart depicting the steps for the preparation of the inorganicorganic precipitated membrane is shown in Fig. 1.

Instruments and membrane characterization

A Rigaku Miniflex X-ray diffractometer was utilized for crystallographic investigation by employing a monochromatic X-ray beam with calcium-filtered $\mathrm{Cu} \mathrm{K} \alpha$ radiation and setting the diffraction angle at between $20^{\circ}$ and $80^{\circ}$. A scanning electron microscope (LEO, $435 \mathrm{VP}$ ) instrument, with gold sputter coatings, operating at $10^{-2}-10^{-3} \mathrm{~Pa}$ with EHT $15.00 \mathrm{kV}$ with $300 \mathrm{~V}$ collector bias was used for the electron micrographs for figuring out the morphological structure of the inorganic-organic nanocomposite membrane. A digital potentiometer model 118 (Electronics India) was used for measuring the membrane potential. 


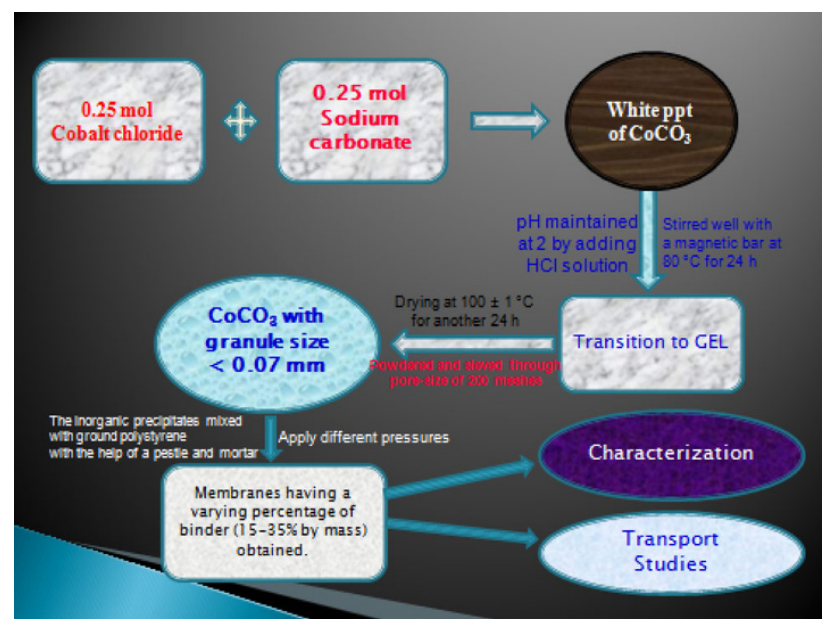

Fig. 1 A flowchart diagram for the fabrication of the composite membrane

Water uptake, volume void porosity and swelling

Different samples of the inorganic-organic nanocomposite membranes prepared with different quantity of polystyrene were immerged in distilled water as well as in $1 \mathrm{M} \mathrm{NaCl}$ solution for $24 \mathrm{~h}$ to figure out water content in terms of water concentration, void porosity and swelling [12, 13]. Their surfaces were wiped with filter paper and then the wet membranes were weighed. The thickness of the samples was measured using a micrometer screw gauge and membrane density for wet membrane was determined by dividing the wet membrane weight by its volume. Subsequently, they were dried at $100{ }^{\circ} \mathrm{C}$ in an oven to constant weight. The weight, thickness and density of dry membranes were also estimated in the same way. Water content and porosity of the membrane were determined in terms of amount of water absorbed by the membrane.

Ion exchange capacity

The ion-exchange capacity (IEC) of the various samples of cobalt carbonate with different amounts of polystyrene was determined by column $(0.5 \mathrm{~cm}$, internal diameter $)$ operation. The ion-exchanger in the $\mathrm{H}^{+}$form was placed in the column with glass wool support; and a $0.1-\mathrm{mol} / \mathrm{L}$ solution of sodium nitrate solution was used as the eluent. The flow rate was maintained at $1.0 \mathrm{~mL} / \mathrm{min}$. The $\mathrm{H}^{+}$ions content of the effluent was then determined by titrating against a standard solution of $0.1 \mathrm{~mol} / \mathrm{L}$ sodium hydroxide.

\section{Chemical stability}

ASTM D543-95 method [14] was used to test the chemical stability of the inorganic-organic nanocomposite membranes. The membranes were exposed to a number of media commonly utilized such as $\mathrm{H}_{2} \mathrm{SO}_{4}, \mathrm{NaOH}, \mathrm{K}_{2} \mathrm{Cr}_{2} \mathrm{O}_{7}, \mathrm{HNO}_{3}$, etc. The membranes were scrutinized after 24,48 and $168 \mathrm{~h}$, analysing variation in colour, texture, splits, holes, bubbles, brightness, decomposition, curving and stickiness.

\section{Measurement of potential}

The freshly prepared inorganic-organic nanocomposite membrane was cemented in a Pyrex glass tube cell having two compartments in which a saturated calomel electrode was placed for measuring the membrane potential; Fig. 2 shows the schematic diagram of the constructed electrochemical cell of the above type. The monovalent chloride electrolytes of concentrations $c_{1}$ and $c_{2}$, in both the compartments of the cell, were vigorously stirred by a magnetic stirrer to minimize the effects of boundary layers on the membrane potential $(\mathrm{mV} \pm 0.5)$. The experiment was conducted at room temperature and atmospheric pressure.

\section{Results and discussion}

Stability of membrane and its physicochemical properties

Our attempt was to set up a membrane system of adequate chemical, thermal and mechanical stability. So, to acquire such membranes, the selection of binder is also of immense importance. Easily available polystyrene is found to be an appropriate binder as its cross-linked rigid framework provides adequate adhesion to the molecules of cobalt carbonate which accounts for the higher mechanical stability to the membrane over other binders like polyvinyl chloride, cellulose acetate, etc. So, a polystyrene blended cobalt carbonate membrane results in more favourable thermal and mechanical stabilities provided by the inorganic part and furnishes good flexibility due to the organic component, i.e. polystyrene-supported cobalt carbonate nanocomposite membrane can be a potential and better candidate than many conventional membranes which degrade under harsh conditions frequently encountered in various industrial settings. Furthermore, it appears to be efficient and cost-effective material having solvent resistance and thermal resistance characteristics [15].

Different amounts of polystyrene (15-35\%) have been blended with the inorganic compound resulting in various blend ratios. The membranes with $25 \%$ organic binder are found to be quite stable and furnish reproducible results; higher or lower than this quantity of polystyrene results in decrease in stability. Membranes prepared in this way did not show any dispersion in water or in other electrolyte solutions. They were subjected to microscopic and electrochemical studies for figuring out cracks and 
Fig. 2 A Schematic representation for the measurement of the membrane potential

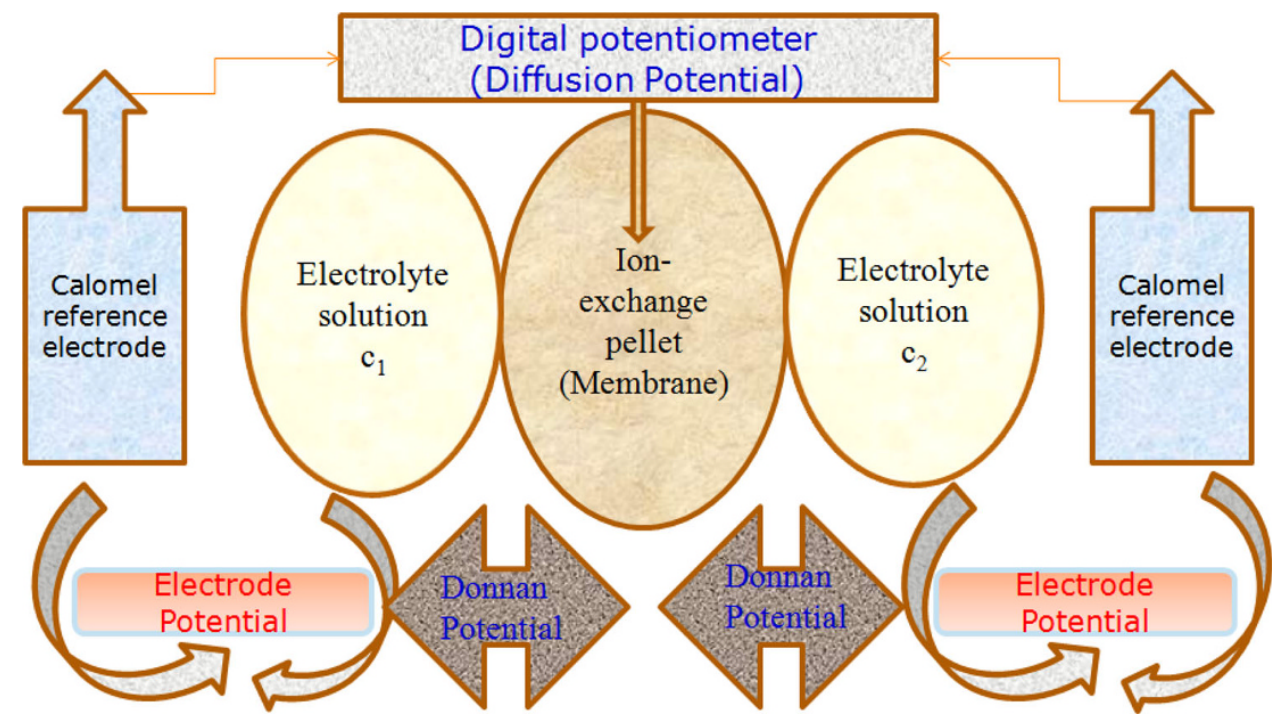

homogeneity of the surface; only those membranes that had smooth surface and generated reproducible potentials were assured by carefully controlling the conditions of fabrication for further studies.

The synthesized inorganic-organic nanocomposite membrane was also tested for chemical resistance in acidic, alkaline and strong oxidant media. In acidic $\left(1 \mathrm{M} \mathrm{H}_{2} \mathrm{SO}_{4}\right)$ and alkaline $(1 \mathrm{M} \mathrm{NaOH})$ media, few considerable changes were observed after 24,48 and $168 \mathrm{~h}$, indicating that the membrane is effective in such media. In strong oxidant media like $\mathrm{K}_{2} \mathrm{Cr}_{2} \mathrm{O}_{7}$, however, the synthesized membrane became fragile after $48 \mathrm{~h}$, losing its mechanical resistance.

Volume fraction of water $\left(\varphi_{\mathrm{w}}\right)$ of the membrane is evaluated by the relation:

$\phi_{\mathrm{w}}=\frac{\Delta \mathrm{w} / \rho_{\mathrm{w}}}{\Delta \mathrm{w} / \rho_{\mathrm{w}}+w_{\mathrm{d}} / \rho_{\mathrm{m}}}$

Here, $\Delta \mathrm{w}\left(=w_{\mathrm{w}}-w_{\mathrm{d}}\right)$ is the weight difference between wet and dry membranes, $\rho_{\mathrm{w}}$ and $\rho_{\mathrm{m}}$ are the densities of water and membrane, respectively. Water content of the pure cobalt carbonate as well as polystyrene-cobalt carbonate samples with different quantities of polystyrene are determined in terms of water concentration (in $\mathrm{mol} / \mathrm{dm}^{3}$ ) in the membrane phase [16] by the following equation:

$W_{\mathrm{c}}^{\mathrm{m}}=\left(\frac{w_{\mathrm{w}}-w_{\mathrm{d}}}{w_{\mathrm{w}} \times M_{\mathrm{W}}}\right) \times \rho_{\mathrm{w}}$,

where $W_{\mathrm{c}}^{\mathrm{m}}$ is the concentration of water in the membrane and $M_{\mathrm{W}}$ is the molar mass of water $(18 \mathrm{~g} / \mathrm{mol})$.

The volume of free water within membrane per unit volume of wet membrane of the composite membrane, defined by Volume void porosity $\left(\tau_{\mathrm{m}}\right)$, is evaluated by the relation [17]

$\tau_{\mathrm{m}}=\frac{\Delta V}{1+\Delta V}$.
Here, $\Delta V$ is the increase in membrane volume upon absorption of the water per unit of dry membrane volume. It is evaluated by the relation $\Delta V=\frac{\left(w_{\mathrm{w}}-w_{\mathrm{d}}\right) \rho_{\mathrm{d}}}{\rho_{\mathrm{w}} w_{\mathrm{d}}}$, where $\rho_{\mathrm{d}}$ is the density of the dry membrane and $\rho_{\mathrm{W}}$ the density of water which enters into the membrane.

The water concentration and volume void porosity of the cobalt carbonate membrane are calculated by Eqs. 2 and 3 and they are plotted as a function of polystyrene in Fig. 3a and $b$; these values are found to decrease with increase in the quantity of polystyrene owing to decrease in interstitial volume. The cross-linked clusters of polystyrene may be the reason for this decrease. All the investigated membranes of cobalt showed a negligibly small swelling when immersed in $\mathrm{NaCl}$ solution for $24 \mathrm{~h}$. The narrow pore size distribution of the membranes increases the diffusive resistance. The low orders of water concentration and volume void porosity with negligible swelling of the membranes also suggest that interstices are negligible and diffusion across the membrane would occur mainly through exchange sites.

Ion-exchange capacity (IEC) (mequiv./g dry memb), which indicates the density of ionisable hydrophilic groups in the membrane matrix and responsible for the ionic conductivity in the ion-exchange membrane, has been calculated from the quantitative analysis of $\mathrm{Na}^{+}$ions by the following equation:

$\mathrm{IEC}=C_{\mathrm{Na}^{+}} V_{\mathrm{sol}} / W_{\text {drymemb }}$,

where $C_{\mathrm{Na}^{+}}$is the concentration of $\mathrm{Na}^{+}$ions $(\mathrm{mmol} /$ $\mathrm{cm}^{3}=$ mequiv. $/ \mathrm{cm}^{3}$ ) of the $\mathrm{NaNO}_{3}$ solution, $V_{\text {sol }}$ the volume of the solution and $W_{\text {drymemb }}$ is the weight of dry membrane $(\mathrm{g})$. The results of the IEC of different samples of cobalt carbonate (Fig. 3c) reveal that the IEC decreases with increase in the mass $\%$ of non-charged polystyrene that 
(a)

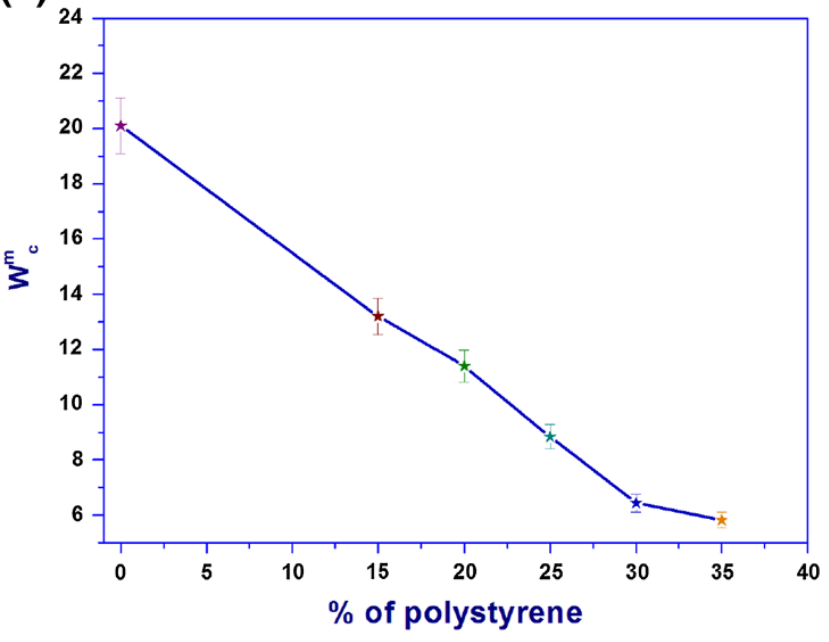

(b)

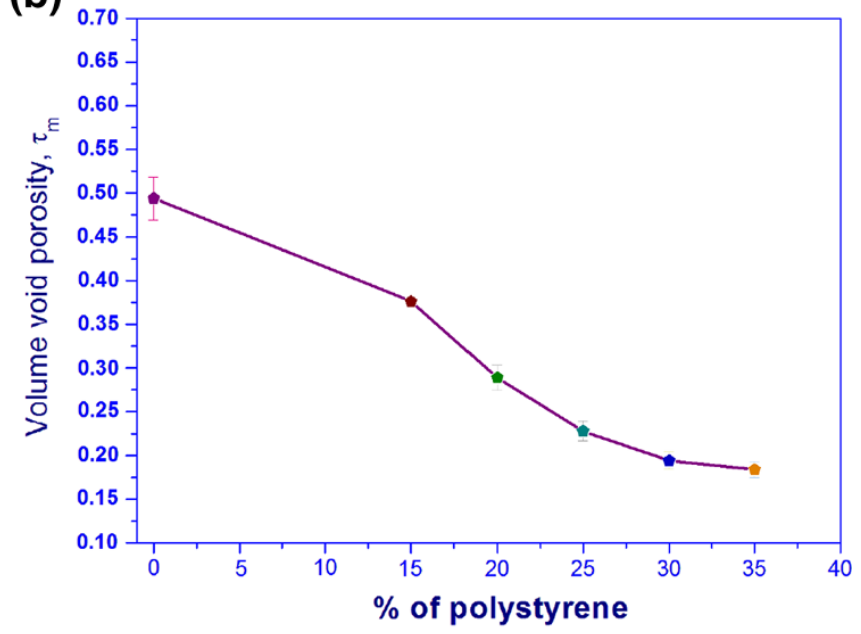

(c)

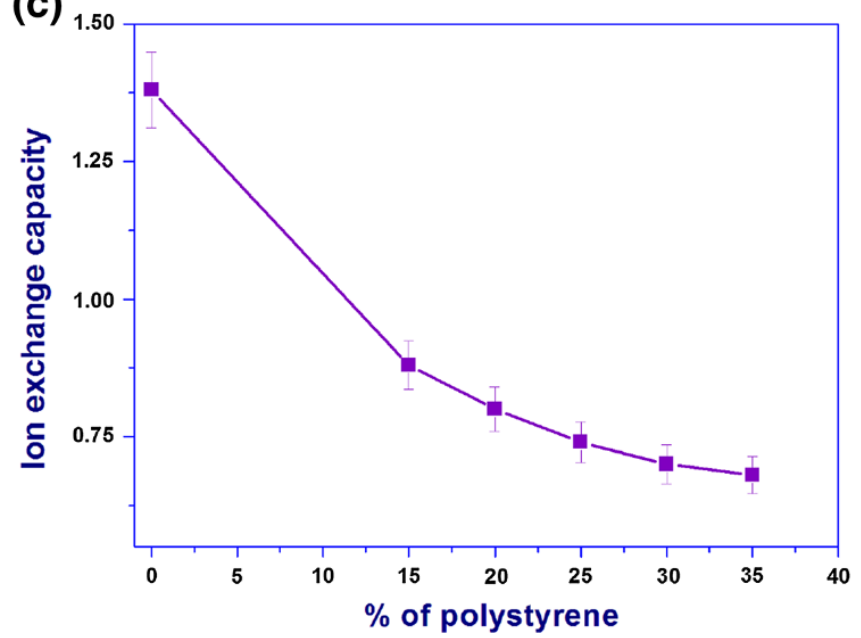

Fig. 3 Plots showing dependence of amount of the organic binder on physicochemical properties: a water concentration, b volume void porosity and $\mathbf{c}$ ion- exchange capacity of cobalt carbonate nanocomposite membrane

is blended with cobalt carbonate. This decrease may also be attributed to the cross-linked clusters of polystyrene. The values of IEC show that cobalt carbonate behaves as a weak cation exchanger.

\section{Characterization}

For a clear demonstration of the surface morphology and the homogeneity in mixing the organic and inorganic substrates to get the hybrid, SEM studies were carried out (Fig. 4). There is no phase separation and cracks of the membrane surface, suggesting that the synthesized composite films are homogeneous in nature and hence form a dense membrane. The surface image of the membrane is shown in Fig. 4a. The porous nature of the membrane is clearly exposed in the image and the membrane is macroscopically uniform in thickness. The pores that can be modelled as uniform capillaries are evenly distributed throughout the surface of the membrane. Entrance and exit effects can be ignored since the pore radius is small as compared to the thickness of the membrane and it is assumed that the membrane and the adjacent solution are in equilibrium. The cross-sectional image of the hybrid membrane is also depicted in Fig. 4b.

X-ray diffractograms (Fig. 5) show the presence of sharp peaks which reveal the crystalline nature of the pure cobalt carbonate and its nanocomposite membranes. The XRD patterns show a broad peak at $31.3^{\circ}$ for pure cobalt carbonate crystal (Fig. 5a) while for the inorganic-organic nanocomposite crystal, the broad peak is observed at $37.2^{\circ}$ (Fig. 5b). It is possible to correlate peak width to the size of crystallographic perpendicular planes using the DebyeScherrer formula: $D=0.9 \lambda / \beta \cos \theta$, which has been used to calculate the crystallite size $(D)$ from the corresponding $\mathrm{X}$-ray spectral peak. Here, $\lambda$ is the $\mathrm{X}$-ray wavelength 
Fig. 4 Scanning electron microscopic a surface and b cross-sectional images of polystyrene blended cobalt carbonate nanocomposite membrane
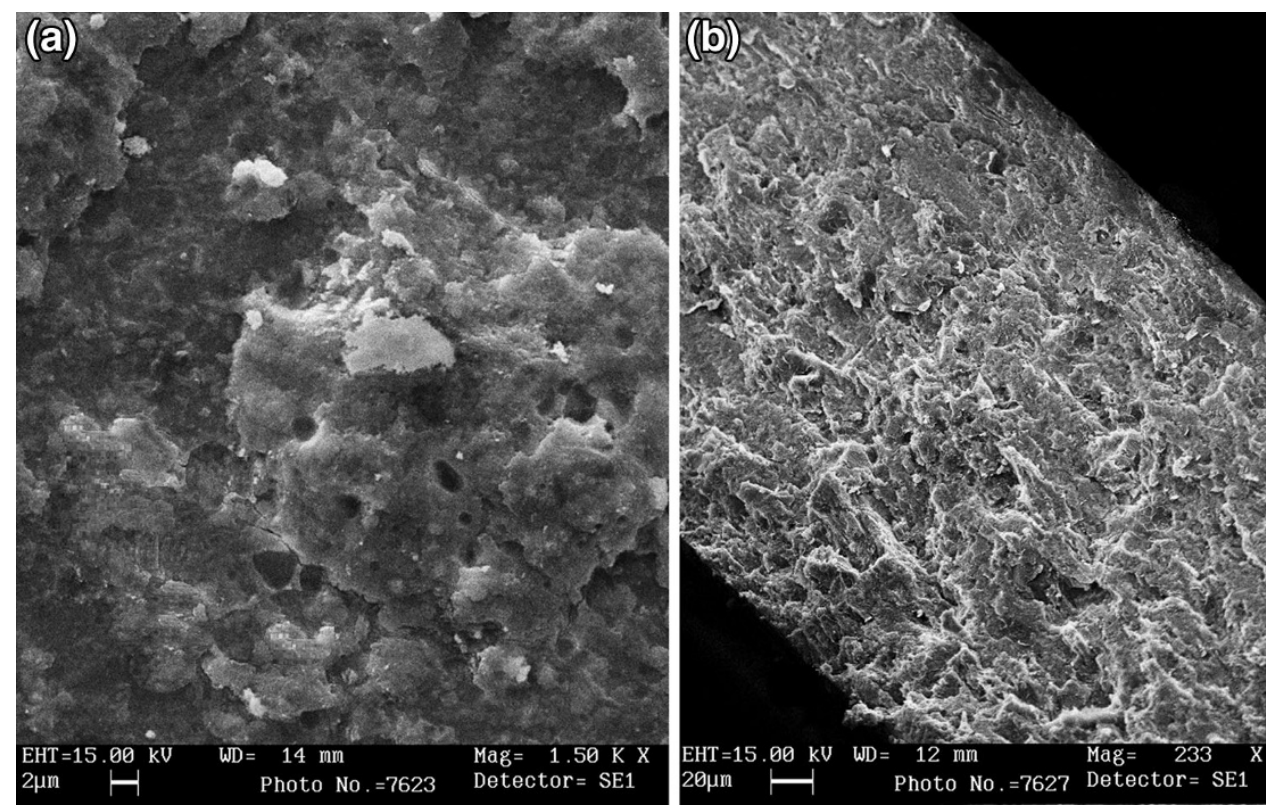

(a)

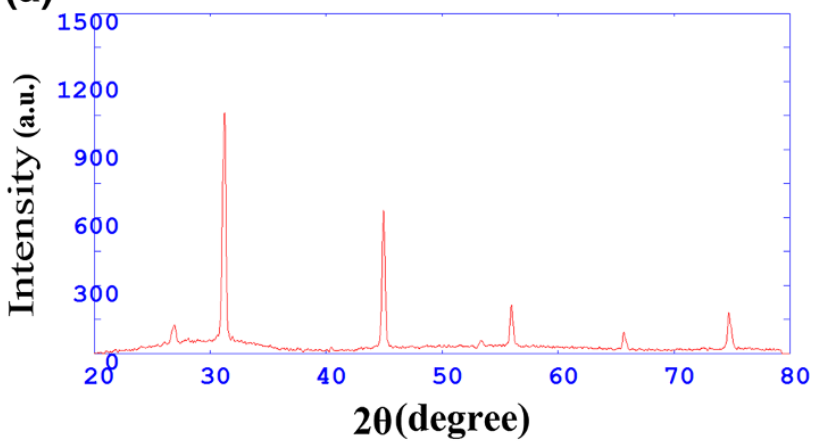

(b)

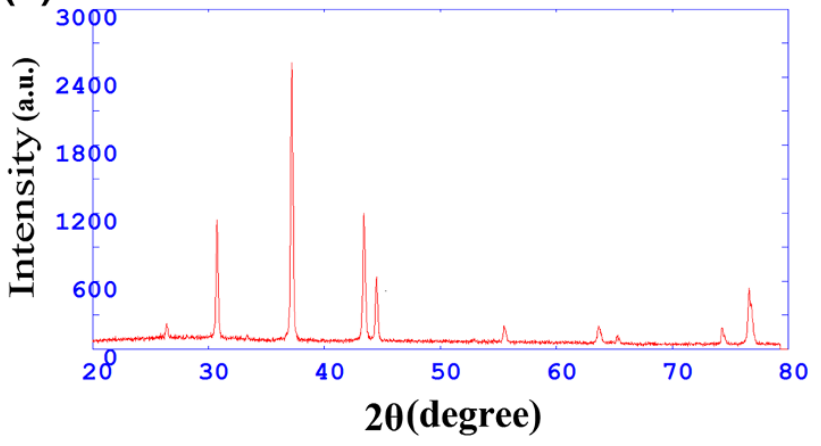

Fig. 5 X-Ray diffractograms of a pure cobalt carbonate and $\mathbf{b}$ polystyrene blended cobalt carbonate membranes

$\left(1.54060 \times 10^{-10} \mathrm{~m}\right)$ of the incident light, $\beta$ is the full width at half-maximum which is the peak width at half of the total peak height of the compound and $\theta$ is the peak diffraction angle. The average crystal granular size of the pure cobalt carbonate nano-particles, calculated from the most intense peak using the above equation, was found to be $22.52 \mathrm{~nm}$ while the crystallite size of the nanocomposite was $35.85 \mathrm{~nm}$. It is also understandable from the diffractograms that the crystalline nature of polystyrene blended cobalt carbonate is less than that of the pure cobalt carbonate; the result is obvious as polystyrene is amorphous in nature [18] which decreases the crystalline nature of cobalt carbonate.

\section{Membrane potential and transport studies}

The membrane potential values $\left(\psi_{\mathrm{m}}\right)$ were measured across the polystyrene blended cobalt carbonate nanocomposite membrane for some monovalent electrolytes of unequal concentrations. The values are plotted as a function of $\log C$, where $C=\left(c_{1}+c_{2}\right) / 2$ is the average concentration with the ratio $\gamma\left(=c_{2} / c_{1}\right)$ fixed at 10 (Fig. 6a). The transport of oppositely charged ions with different mobilities develops a potential difference across the membrane. The quantity of charge necessary to generate the potential is small, particularly when dilute solutions are used. The charge imparts some significant electrochemical properties to the membrane such as the differences in the permeabilities of co-ions, counter ions and neutral molecules. The magnitude of the membrane potential depends on many factors like applied pressure at the membrane preparation stage, counter-ion to co-ion mobility ratio, exchange characteristics of the membrane material for various cations in addition to the nature and concentration of the equilibrating electrolyte solutions [19]. It was observed that the cobalt carbonate nanocomposite membrane prepared at higher applied pressure exhibited higher membrane potential. The results reveal that the potential 
(a)

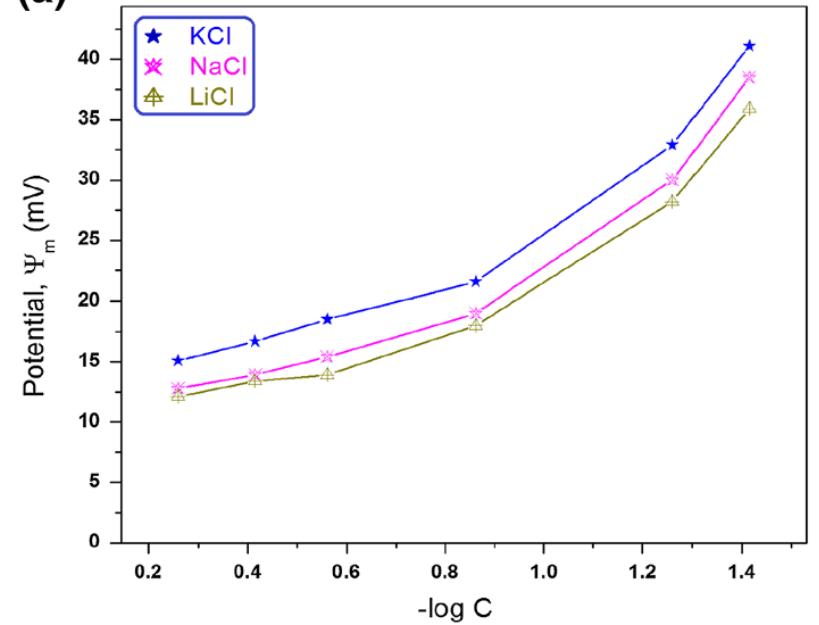

(b)

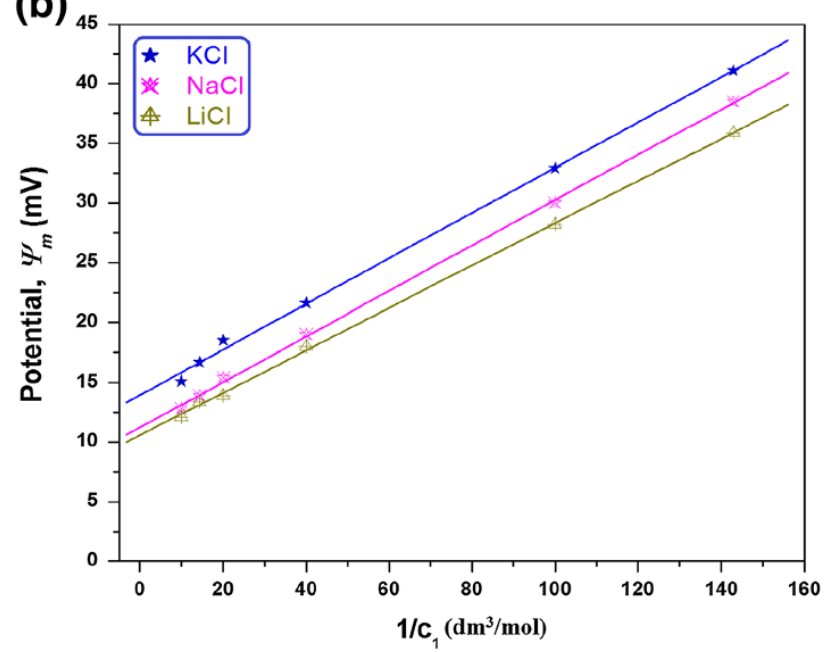

(c)

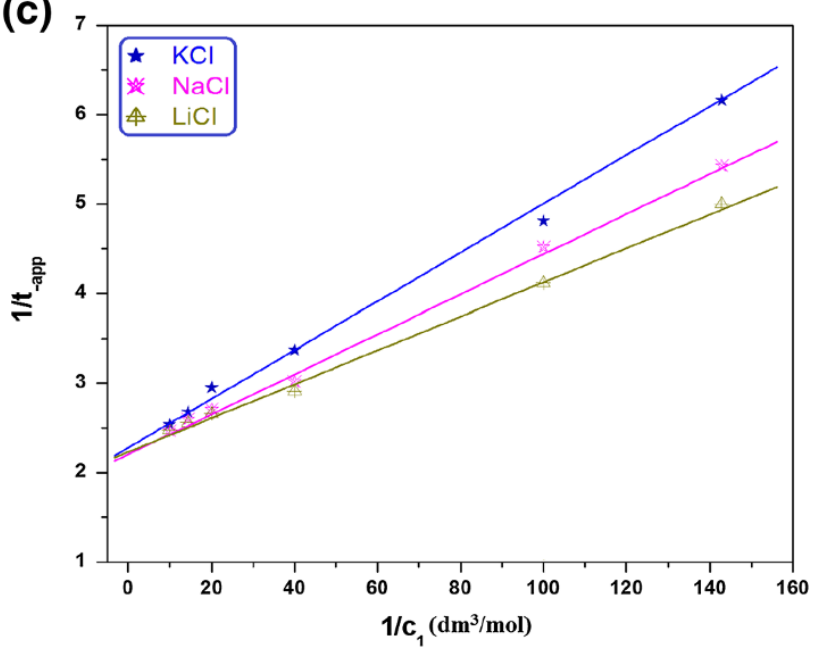

Fig. 6 Plots showing a membrane potentials, $\psi_{\mathrm{m}}$ against $-\log C$, b potential values, $\psi_{\mathrm{m}}$ against $1 / c_{1}$ and $\mathbf{c} 1 / t_{\text {-app }}$ against $1 / c_{1}$ across cobalt carbonate nanocomposite membrane equilibrated with various monovalent electrolytes at an applied pressure of $146 \mathrm{MPa}$ values follow the order $\mathrm{K}^{+}>\mathrm{Na}^{+}>\mathrm{Li}^{+}$. The membrane potential was observed to increase with time at first, attained a maximum value after a certain interval and then fell off slowly. For a concentrated solution, the time taken for the attainment of maximum potential was observed to be more than that of a dilute solution but it was found to differ with different electrolytes.

The measurement of ion activity by means of a membrane electrode is most successful in the concentration range over which the membrane behaves as ideally permselective and obeys the Nernst equation. An ideally permselective membrane is the one which allows a negligible permeability for co-ions as compared to that for counter-ions. The co-ion transference and the dependence of the exchange of cations between the solution and the membrane phase and on the electrolyte concentration [20] are possibly the reason for the deviation. The data in Fig. 6a illustrate that the potential values are positive and increase with decrease in concentration of all the tested electrolytes, which shows that the membrane is negatively charged, i.e. cation selective as cations easily pass through the negatively charged cation exchange membrane. The overall outcome of the process is that one cell of the pair becomes depleted of ions while the adjacent cell becomes enriched in ions; however, the selectivity increases with dilution which may be due to the structural alteration produced in the electrical double layer at the membranesolution interface. The increase in selectivity with dilution is also supported by the increasing values of the counterion transport numbers, $t_{+}$(Table 1).

The influence of penetration of mobile species into the membrane when an ionic gradient is maintained by two solutions of an electrolyte of different concentrations on both sides of the membrane is greater in case of counter-ions than in the co-ion. The values of the ratio of the molar mobilities of the cation and anion ' $u_{+} / u_{-}$' (the mobility ratio $(\bar{\omega})$ of the membrane) calculated for cobalt carbonate nanocomposite membrane are also represented in Table 1 . The values of $\bar{\omega}$ in the membrane phase were found to be increasing with dilution for all the monovalent electrolytes used. The high mobility is attributed to higher transport number of comparatively free cations as compared to the anion $\left(\mathrm{Cl}^{-}\right.$ion) of the electrolytes.

When a slightly charged membrane was imposed between two solutions of an electrolyte of unequal concentrations $c_{1}$ and $c_{2}\left(c_{2}>c_{1}\right)$ with the effective concentration of ion-exchange site of the membrane much lower than electrolyte concentration $(C)$, the following equation was derived for the potential developed across the membrane [19]: 
Table 1 Values of transport number $\left(t_{+}\right)$and mobility ratio $(\bar{\omega})$ calculated for cobalt carbonate nanocomposite membrane from the measured membrane potential values with various monovalent electrolytes at different concentrations with $c_{2} / c_{1}=10$ at an applied pressure $146 \mathrm{MPa}$ at $25 \pm 1{ }^{\circ} \mathrm{C}$

\begin{tabular}{llll}
\hline Electrolyte & $c_{2}\left(\mathrm{~mol} / \mathrm{dm}^{3}\right)$ & $t_{+}$ & $\bar{\omega}$ \\
\hline $\mathrm{KCl}$ & 1 & 0.628 & 1.689 \\
& 0.7 & 0.641 & 1.787 \\
& 0.5 & 0.656 & 1.910 \\
& 0.25 & 0.682 & 2.149 \\
& 0.1 & 0.778 & 3.505 \\
& 0.07 & 0.848 & 5.559 \\
$\mathrm{NaCl}$ & 1 & 0.609 & 1.555 \\
& 0.7 & 0.617 & 1.613 \\
& 0.5 & 0.630 & 1.705 \\
& 0.25 & 0.661 & 1.948 \\
& 0.1 & 0.754 & 3.067 \\
& 0.07 & 0.826 & 4.741 \\
$\mathrm{LiCl}$ & 1 & 0.602 & 1.513 \\
& 0.7 & 0.613 & 1.584 \\
& 0.5 & 0.617 & 1.613 \\
& 0.25 & 0.652 & 1.874 \\
& 0.1 & 0.739 & 2.830 \\
& 0.07 & 0.804 & 4.104 \\
& & & \\
& & &
\end{tabular}

$\psi_{\mathrm{m}}=(\mathrm{RT} / \mathrm{F})\left[\left(2 t_{+}^{\mathrm{m}}-1\right) \ln \gamma+\frac{2(\gamma-1)}{\gamma}\left(1-t_{+}^{\mathrm{m}}\right) t_{+}^{\mathrm{m}} \frac{X_{\mathrm{f}}}{c_{1}}\right]$,

where $X_{\mathrm{f}}$ is the membrane fixed charge concentration and $t_{+}^{\mathrm{m}}$ is the counter-ion transport number in the membrane phase. Equation (5) represents a linear relationship between $\psi_{\mathrm{m}}$ and $1 / c_{1}$ (Fig. 6b) which allows the evaluation of $X_{\mathrm{f}}$ and $t_{+}^{\mathrm{m}}$ values of the nanocomposite membrane from the slope and intercept for different electrolytes (Table 2). The $t_{+}^{\mathrm{m}}$ values are found to be proportional to the values of $X_{\mathrm{f}}$, i.e. both are found to increase with decrease in electrolyte concentration. The results show that the counter-ion transport numbers follow the order $\mathrm{K}^{+}>\mathrm{Na}^{+}>\mathrm{Li}^{+}$in the carbonate membrane. The calculated values also reveal that the nanocomposite membrane exhibits moderate selectivity towards all cations and that for the same concentration, the selectivity of the investigated carbonate membrane is comparatively more towards $\mathrm{K}^{+}$ions as selectivity is proportional to counter-ion transport number, as suggested by the values given in the table.

Tasaka et al. [8] suggested the following relation to calculate fixed charge density of the membrane:

$-\psi_{\mathrm{m}}=\frac{\mathrm{RT}}{F}\left(\frac{\gamma}{\gamma-1}\right)\left(\frac{\phi X}{2}\right) \frac{1}{c_{1}}$.
Table 2 The values of the calculated effective fixed charge densities, from different approaches, of the cobalt carbonate nanocomposite membrane in contact with different electrolytes with their counter-ion transport number, $t_{+}^{\mathrm{m}}$ in the membrane phase

\begin{tabular}{llll}
\hline Electrolyte & $\mathrm{KCl}$ & $\mathrm{NaCl}$ & $\mathrm{LiCl}$ \\
\hline$X_{\mathrm{f}}$ & 0.0165 & 0.0160 & 0.0138 \\
$\phi X$ & 0.0126 & 0.0119 & 0.0108 \\
$\theta \overline{X_{\mathrm{c}}}$ & 0.0541 & 0.0468 & 0.0372 \\
$\theta \bar{X}$ & 0.0621 & 0.0566 & 0.0487 \\
$t_{+}^{\mathrm{m}}$ & 0.6179 & 0.5949 & 0.5890 \\
\hline
\end{tabular}

Here, $\phi X$ is the effective fixed charge density of the negatively charged membrane. Equation 6 indicates that the plot of $\psi_{\mathrm{m}}$ against $1 / c_{1}$ will be linear. The plot shown in Fig. $6 \mathrm{~b}$ has been used but with a different slope equal to $\frac{\mathrm{RT}}{F}\left(\frac{\gamma}{\gamma-1}\right)\left(\frac{\phi X}{2}\right)$ from which the values of $\phi X$ for different electrolytes have been evaluated (Table 2).

It was also shown, at the same experimental condition, that the inverse of the apparent transport number of the anion, $t_{\text {-app }}$, in a high salt concentration range could be expressed by the following equation [21]:

$\frac{1}{t_{- \text {app }}}=\frac{1}{1-\alpha}+\frac{\alpha(\gamma-1)}{(1-\alpha) \gamma \ln \gamma}\left(\frac{\theta \overline{X_{\mathrm{c}}}}{c_{1}}\right)$,

Here, $\alpha$ is the ratio of molar mobility of cation to the sum of molar mobilities of cation and anion, $\theta \overline{X_{c}}$ is the effective fixed charge density of the membranes under investigation and $c_{1}$ (in mol/ $\mathrm{dm}^{3}$ ) the concentration of the monovalent electrolyte in the lower concentration side of the cell. The apparent transport number of the anion $t_{\text {-app }}$ is defined by the Nernst equation: $\psi_{\mathrm{m}}=(\mathrm{RT} / F)$ $\left(1-2 t_{- \text {app }}\right) \ln \frac{c_{2}}{c_{1}}$. Equation (7) shows that the values of $\alpha$ and $\theta \overline{X_{\mathrm{c}}}$ can be evaluated from the values of intercept $\left(\frac{1}{1-\alpha}\right)$ and slope $\left(\frac{\alpha(\gamma-1)}{(1-\alpha) \gamma \ln \gamma} \theta \overline{X_{\mathrm{c}}}\right)$ from the linear plot of $1 / t_{\text {-app }}$ against $1 / c_{1}$ (Fig. 6c). The calculated values of the fixed charge densities $\theta \overline{X_{\mathrm{c}}}$ of the polystyrene blended cobalt carbonate composite membrane are also given in Table 2 which unveils the order $\mathrm{KCl}>\mathrm{NaCl}>\mathrm{LiCl}$.

The term, permselectivity $P_{\mathrm{s}}$ is defined as a measure of preferential permeation of counter-ions inside the membrane as compared to solution outside the membrane, and ion selectivity of an ion-exchange membrane can also be expressed as a function of it. Applying approach proposed by Helfferich [22], permselectivity has been evaluated by the following equation: $P_{\mathrm{s}}=\left(t_{+}^{\mathrm{m}}-t_{+}\right) /\left(1-t_{+}\right)$, where $t_{+}^{\mathrm{m}}$ and $t_{+}$are the true transport numbers of the counter-ion in the membrane and solution, respectively. The permselectivity arises because of the nature of the membrane for inequity between counter-ions and co-ions. Such type of 


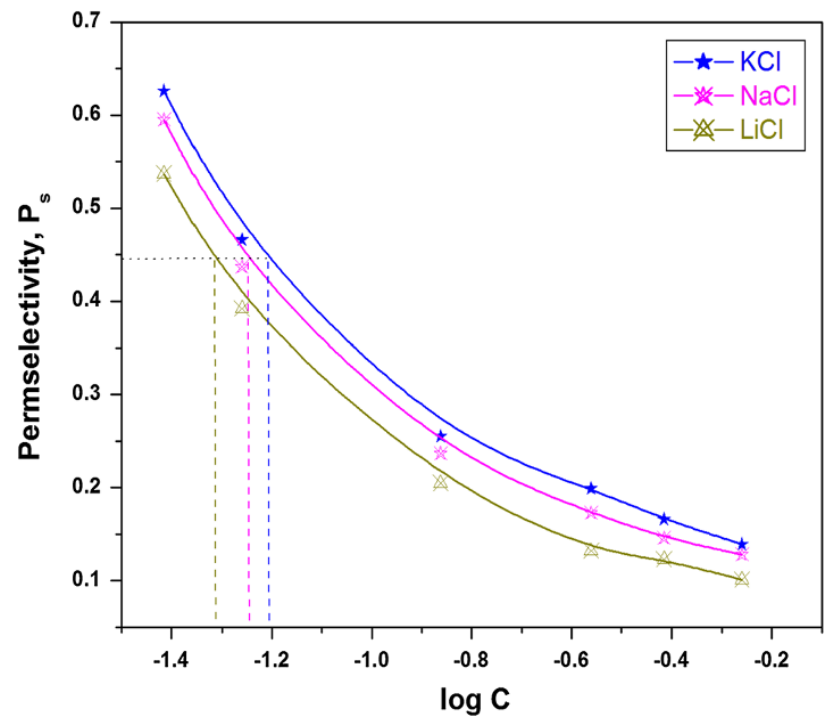

Fig. 7 Permselectivity $\left(P_{\mathrm{s}}\right)$ against $\log C$ for polystyrene-based cobalt carbonate membrane with different monovalent electrolytes

discrimination arises as a result of the nature and magnitude of the charge, the so-called concentration of fixed charge on membrane surface $\left(X_{\mathrm{f}}\right)$ that is associated with the membrane matrix. $X_{\mathrm{f}}$ can be expressed in terms of permselectivity, $P_{\mathrm{s}}$ by the relation: $X_{\mathrm{f}}=\frac{2 \mathrm{CP}_{\mathrm{s}}}{\sqrt{1-P_{\mathrm{s}}^{2}}}$ [23]. The calculated values of $P_{\mathrm{s}}$ are plotted against $\log C$ of the polystyrene blended cobalt carbonate membrane in contact with various monovalent electrolytes (Fig. 7). The decline in permselectivity values with the increase in electrolyte concentration may be due to the reduction of the Donnan exclusion. It was also suggested that the fixed charge density of a membrane could be calculated from the data of permselectivity $[9,10]$.

$\frac{1}{\sqrt{4 \xi^{2}+1}}=\frac{1-t_{-\mathrm{app}}-\alpha}{\alpha-(2 \alpha-1)\left(1-t_{-\mathrm{app}}\right)} \equiv P_{\mathrm{s}}$

where $\xi=\frac{C}{\theta \bar{X}}$.

Here, the product $\theta \bar{X}$ is termed as the thermodynamically effective fixed charge density of the membrane and $P_{\mathrm{s}}$ is the permselectivity of the membrane-electrolyte system the value of which can also be calculated from the membrane potential data by inserting the values of $\alpha$ and $t_{\text {-app }}$. Equation (8) indicates that if $P_{\mathrm{s}}=1$, then the transport number of the co-ions $\left(t_{\text {-app }}\right)$ is zero, i.e. the membrane is perfectly selective; while if $P_{\mathrm{s}}=0$, then $t_{\text {-app }}=1-\alpha$, i.e. anions behave as in the bulk solution or as in a membrane having no fixed charge. It is evident from the left-hand side of equation that when $C$ becomes equal to $\theta \bar{X}, P_{\mathrm{s}}$ attains the value of $1 / \sqrt{5}$ or 0.447 . The value of $C$ corresponding to which $P_{\mathrm{s}}=0.447$ will give the value of the fixed charge density $(\theta \bar{X})$. The values of the fixed charge densities calculated from the plots of $P_{\mathrm{s}}$ against $\log C$ (Fig. 7) for various cobalt carbonate membrane-electrolyte systems are also included in Table 2. The results provide information that the fixed charge density is highest for $\mathrm{LiCl}$ and lowest for $\mathrm{NaCl}$ for the same electrolytic concentration, indicating that the polystyrene incorporated cobalt carbonate nanocomposite membrane shows highest selectivity towards $\mathrm{K}^{+}$ ions.

\section{Conclusion}

The nanocomposite membrane prepared with $25 \%$ polystyrene provides reasonably higher chemical and mechanical stabilities of cobalt carbonate nanocomposite membrane in water and produces fine results for electrochemical purposes and hence can be a potential and better contender than many conventional membranes which degrade under harsh conditions often encountered in various industrial applications. The physicochemical properties (water content and volume void porosity) of cobalt carbonate composite membrane are found to decrease with increase in the percentage of polystyrene. The inorganic-organic composite membrane behaves as a weak cation-exchanger and its exchange capacity decreases abruptly with increasing amount of polystyrene. The counter-ion transport numbers, permselectivity and effective fixed charge densities are in the order $\mathrm{KCl}>\mathrm{NaCl}>\mathrm{LiCl}$ for the same electrolytic concentration which indicates that the composite membrane shows higher selectivity towards $\mathrm{Li}^{+}$ions. Moreover, the values of fixed charge densities calculated by different methods (Table 2) are found to be in close agreement. Characterization by SEM and X-ray analyses reveal the morphological structure and the crystalline nature of the nanocomposite cation-exchange membrane.

Acknowledgments We are grateful to the University Grant Commission (UGC), New Delhi, for financial assistance for our research work. We also express our great appreciation to All India Institute of Medical Sciences (AIIMS), New Delhi, for providing SEM images and Department of Applied Physics, Aligarh Muslim University (AMU) for X-ray diffractograms.

Open Access This article is distributed under the terms of the Creative Commons Attribution License which permits any use, distribution, and reproduction in any medium, provided the original author(s) and the source are credited.

\section{References}

1. Schaep, J., Vandecasteele, C.: Evaluating the charge of nanofiltration membranes. J. Membr. Sci. 188, 129-136 (2001)

2. Fievet, P., Aoubiza, B., Szymczyk, A., Pagetti, J.: Membrane potential in charged porous membranes. J. Membr. Sci. 160, 267-275 (1999) 
3. Lakashminarayanaiah, N.: Membrane electrode. Academic Press, New York (1962)

4. Hickner, M.A., Ghassami, H., Kim, Y.S., Einsla, B.R., McGrath, J.E.: Alternative polymer systems for proton exchange membranes (PEMs). Chem. Rev. 104, 4587-4612 (2004)

5. Nagarale, R.K., Gohil, G.S., Shahi, V.K., Rangarajan, R.: Organic-inorganic hybrid membranes: thermally stable cationexchange membrane by sol-gel method. Macromolecules 37, 10023-10030 (2004)

6. Ganesan, V., Walcarius, A.: Surfactant templated sulfonic acid functionalized silica microspheres as efficient ion exchangers and electrode modifiers. Langmuir 20, 3632-3640 (2004)

7. Chung, T., Jiang, L.Y., Li, Y., Kulprathipanja, S.: Mixed matrix membranes (MMMs) comprising organic polymers with dispersed inorganic fillers for gas separation. Prog. Polym. Sci. 32, 483-507 (2007)

8. Tasaka, M., Aoki, N., Kondo, Y., Nagasawa, M.: Membrane potentials and electrolyte permeation velocities in charged membranes. J. Phys. Chem. 79, 1307-1314 (1975)

9. Kobatake, Y., Takeguchi, N., Toyoshima, Y., Fujita, H.: Studies of membrane phenomena: I. Membrane potential. J. Phys. Chem. 69, 3981-3988 (1965)

10. Kamo, N., Oikawa, M., Kobatake, Y.: Effective fixed charge density governing membrane phenomena. V. A reduced expression of permselectivity. J. Phys. Chem. 77, 92-95 (1973)

11. Khan, M.R., Rafiuddin, : Synthesis, characterization and properties of polystyrene incorporated calcium tungstate membrane and studies of its physicochemical and transport behaviour. Desalination 329, 103-114 (2013)

12. Mizutani, Y., Nishimura, M.: Studies on ion-exchange membranes. XXXII. Heterogeneity in ion-exchange membranes. J. Appl. Polym. Sci. 14, 1847 (1970)

13. Nagarale, R.K., Gohil, G.S., Shahi, V.K., Rangarajan, R.: Preparation of organic-inorganic composite anion-exchange membranes via aqueous dispersion polymerization and their characterization. J. Colloid Interface Sci. 287, 198-206 (2005)

14. ASTM D543-95, Standard particles for evaluating the resistance of plastics to chemical reagents, pp. 27-33 (1998)
15. Razdan, U., Joshi, S.V., Shah, V.J.: Novel membrane processes for separation of organics. Curr. Sci. 85, 761-771 (2003)

16. Reddy, R.N., Reddy, R.G.: Electrochemical double layer capacitance properties of carbon in aqueous and non-aqueous electrolytes. J. Power Sources 132, 315-320 (2004)

17. Capeci, S.W., Pintauro, P.N., Bennion, D.N.: The molecular-level interpretation of salt uptake and anion transport in nafion membranes. J. Electrochem. Soc. 136, 2876-2882 (1989)

18. Boccaleri, E., Arrais, A., Frache, A., Gianelli, W., Fino, P., Camino, G.: Comprehensive spectral and instrumental approaches for the easy monitoring of features and purity of different carbon nanostructures for nanocomposite applications. Mat. Sci. Eng. B Solid 131, 72-82 (2006)

19. Gohil, G.S., Shahi, V.K., Rangarajan, R.: Comparative studies on electrochemical characterization of homogeneous and heterogeneous type of ion-exchange membranes. J. Membr. Sci. 240, 211-219 (2004)

20. Siddiqi, F.A., Khan, I.R., Saksena, S.K., Ahshan, M.A.: Studies with model membranes. : XI. Evaluation of thermodynamic parameters for diffusion and fixed charge density by methods based on thermodynamics of irreversible processes. J. Membr. Sci. 2, 245-261 (1977)

21. Khan, H.R., Ozeki, S.: Estimation of effective fixed charge density of a black lipid membrane of didodecyl phosphite from membrane potential and its application to static magnetic field effect on ion transport. J. Colloid Interface Sci. 177, 628-632 (1996)

22. Helfferich, F.G.: Ion exchange. Dover Publication, New York (1962)

23. Shahi, V.K., Trivedi, G.S., Thampy, S.K., Rangarajan, R.: Studies on the electrochemical and permeation characteristics of asymmetric charged porous membrane. J. Colloid Interface Sci. 262, 566-573 (2003) 